Revue

Revue de l'histoire des religions

de Ihistoire des religions

Jean-Robert ARMOGATHE, Histoire des idées religieuses et scientifiques dans l'Europe moderne. Quarante ans d'enseignement à l'École Pratique des Hautes Études Turnhout, Brepols, (« Bibliothèque de l'École Pratique des Hautes Études »), 2012

Xavier Kieft

\title{
OpenEdition
}

Journals

Édition électronique

URL : http://journals.openedition.org/rhr/8627

DOI : $10.4000 /$ rhr.8627

ISSN : 2105-2573

Éditeur

Armand Colin

Édition imprimée

Date de publication : 1 septembre 2016

Pagination : 468-469

ISBN : 978-2-200-93061-5

ISSN : 0035-1423

Référence électronique

Xavier Kieft, « Jean-Robert armogathe, Histoire des idées religieuses et scientifiques dans l'Europe moderne. Quarante ans d'enseignement à l'École Pratique des Hautes Études », Revue de l'histoire des religions [En ligne], 3 | 2016, mis en ligne le 06 octobre 2016, consulté le 25 septembre 2020. URL http://journals.openedition.org/rhr/8627 ; DOI : https://doi.org/10.4000/rhr.8627

Ce document a été généré automatiquement le 25 septembre 2020.

Tous droits réservés 


\section{Jean-Robert ARMOGATHE, Histoire des idées religieuses et scientifiques dans l'Europe moderne. Quarante ans d'enseignement à l'École Pratique des Hautes Études}

Turnhout, Brepols, (« Bibliothèque de l'École Pratique des Hautes

Études »), 2012

Xavier Kieft

\section{RÉFÉRENCE}

Jean-Robert ARMOGATHE, Histoire des idées religieuses et scientifiques dans l'Europe moderne. Quarante ans d'enseignement à l'École Pratique des Hautes Études, Turnhout, Brepols, («Bibliothèque de l'École Pratique des Hautes Études »), 2012, 23,4 cm, 219 p., $50 €$, ISBN 978-2-503-54488-5.

Le présent ouvrage porte le nom de la chaire occupée à partir de 1991, à la section des Sciences religieuses de l'École Pratique des Hautes Études, par Jean-Robert Armogathe. Il fut d'abord chargé de conférences, puis maître de conférences dans ce même établissement où il effectua l'essentiel de sa carrière universitaire principale depuis 1970 - il a également assuré des enseignements dans plusieurs autres universités (p.11). Ce volume réunit les comptes rendus de quarante années de séminaires, une vaste bibliographie raisonnée (p. 181-195) et un indispensable index permettant de se repérer de manière transversale dans le volume, faisant de celui-ci un véritable outil de travail et non le simple témoin d'une carrière doublement exceptionnelle: par sa longévité, bien sûr, mais aussi par l'exemplaire fécondité de la recherche qui y fut mise en œuvre. Les comptes rendus originellement parus dans l'Annuaire de la section des 
sciences religieuses sont augmentés de quelques notes et d'utiles renvois aux parutions figurant dans la bibliographie auxquelles les travaux menés ont pu donner lieu.

2 Le lecteur curieux découvre ainsi un impressionnant réseau de recherches constitué par les entrecroisements d'axes distincts, mais jamais exclusifs les uns des autres, dont les principaux sont l'histoire du cartésianisme, l'histoire religieuse, notamment celle des controverses, de Port Royal et du jansénisme, des explications eucharistiques, de l'exégèse, mais aussi des idées scientifiques, sans oublier la lexicographie et l'histoire des concepts philosophiques, théologiques et scientifiques, principalement aux XvII ${ }^{\mathrm{e}}$ $\mathrm{XVIII}^{\mathrm{e}}$ siècles. Le chercheur prendra la mesure d'une méthode de travail remarquable en histoire des idées, qui s'inscrit, comme l'indique le titre donné par J.-R. Armogathe à ses recherches, dans le prolongement des intuitions d'Alexandre Koyré dont la chaire à l'EPHE s'intitulait "Histoire des idées religieuses dans l'Europe moderne ». En reprenant ce titre, mais en ajoutant «et scientifiques», J.-R. Armogathe a souligné l'articulation, indiquée par Koyré, des concepts théologiques et scientifiques, les uns ne trouvant pas leur signification indépendamment des autres. Son travail consiste dès lors à montrer que leur importance s'éprouve dans les controverses, dans les échanges savants et les correspondances, dans la réception et la stimulation des découvertes en physiques, mais également dans les théories politiques et les perspectives morales qu'elles appellent ou rendent possibles.

3 Appuyée sur une appréhension souvent exhaustive des textes (l'influence de la méthode quantitative en histoire est perceptible dans les premiers comptes rendus), la recherche a impliqué le développement d'une érudition remarquable, jusque dans la lecture et la redécouverte d'auteurs réputés si mineurs que J.-R. Armogathe les a souvent lui-même tirés de l'oubli des archives et des bibliothèques. Une rapide consultation de l'index permettra à chacun de prendre la mesure de sa propre ignorance d'un grand nombre des figures auxquelles le chercheur a su accorder l'importance qui leur revenait. C'est qu'en matière d'histoire des idées, il n'existe pas réellement d'auteur mineur, et la juste interprétation des positions et des apports de chacun ne peut s'établir qu'en étant rapportée au contexte réel dans lequel ils sont apparus: contexte pour lequel l'histoire des grands mouvements de pensée n'a pas encore opéré la sélection des quelques jalons que l'on estime, de manière commode mais souvent bien grossière et inexacte, particulièrement révélateurs.

C'est pourquoi le lecteur du présent livre sera bien en peine de retracer un cheminement linéaire à la progression marquée. La recherche s'est au contraire établie comme l'exploration attentive, continue et répétée du vaste domaine des idées modernes et la reconstitution de leur circulation et de leur évolution. L'attention prêtée aux renvois bibliographiques sera à ce titre éclairante : on remarque que si les travaux de J.-R. Armogathe font parfois l'objet d'une maturation de nombreuses années avant de prendre la forme d'un texte publié, ses articles et ses ouvrages peuvent également faire l'objet d'approfondissements bien des années après leur parution sous une première forme. En effet, la quantité des pistes dégagées s'avère saisissante. Si la bibliographie scientifique présentée est imposante, elle ne recouvre qu'une partie des recherches menées au sein de l'EPHE. Au reste, ceux qui ont assisté à ces conférences savent à quel point les comptes rendus de l'Annuaire sont lacunaires par rapport au travail effectivement mené lors des séances du vendredi matin. Et si l'on considère le nombre de cours et de séminaires dispensés par ailleurs, sur des thèmes souvent différents de ceux de la chaire en question, on s'aperçoit que le présent ouvrage n'offre 
encore qu'un reflet parcellaire des apports de J.-R. Armogathe à la culture et à l'érudition contemporaines.

5 Quoi qu'il en soit, en indiquant quelques-unes des pistes déjà empruntées et en renvoyant aux textes scientifiques - toujours d'une grande concision - dans lesquels certains parcours ont pu être consignés, ce livre se donne comme l'outil indispensable de celui qui veut entreprendre ou prolonger des études relatives à cette histoire des idées religieuses et scientifiques. Rappelons pour finir, à l'attention de celui qui voudrait prendre connaissance d'un itinéraire personnel et intellectuel auquel la retraite de l'EPHE n'a nullement mis un terme, la parution chez Calmann-Lévy en 1991 d'un livre d'entretiens avec Jean Lebrun, intitulé Raison d'Église. De la rue d'Ulm à NotreDame.

\section{AUTEURS}

\section{XAVIER KIEFT}

Université Paris-Sorbonne. 